\title{
SITIOS DEL VALLE DE MÉXICO EN LOS QUE SE DEBE DISEÑAR PARA UN Q MENOR AL ESTIPULADO PARA ESTRUCTURAS DÚCTILES
}

\author{
José E. Barradas Hernández ${ }^{(1)}$ y Gustavo Ayala Milián ${ }^{(2)}$
}

\begin{abstract}
RESUMEN
Se muestra que a manera de que las estructuras que se ubicarán sobre suelos del valle de México con periodos dominantes mayores a $1.65 \mathrm{~s}$ y diseñadas con lineamientos del apéndice A de las NTCS- 2004, cumplan los parámetros de desempeño establecidos, deben diseñarse para un valor máximo del factor de comportamiento sísmico $Q$; deducido en el presente estudio, que es menor a los estipulados para estructuras dúctiles. Para ilustrar lo anterior, se diseñan dos marcos de concreto reforzado: uno para un $Q=4$ y otro para el valor máximo de $Q$. Se demuestra que el marco diseñado para $Q=4$ responde inelásticamente al sismo de diseño asociado al estado límite de servicio, y por tanto que no cumple los parámetros de desempeño requeridos. También se demuestra que el marco diseñado para el valor máximo de $Q$, al responder elásticamente al sismo de diseño asociado al estado límite de servicio, cumple los parámetros de desempeño establecidos.
\end{abstract}

Palabras clave: factor de sobrerresistencia; factor de comportamiento sísmico; diseño sísmico multinivel de edificios

\section{SITES OF THE VALLEY OF MEXICO IN WHICH A VALUE OF Q FOR DESIGN LOWER THAN THAT STIPULATED FOR DUCTILE STRUCTURES MUST BE CONSIDERED}

\begin{abstract}
It is shows that in order of that the structures to be located on soils of the valley of Mexico with dominant periods higher than $1.65 \mathrm{~s}$, and designed according guidelines to Appendix $\mathrm{A}$ of the NTCS- 2004, meets the established performance parameters, they must be designed for a maximum value of seismic behavior factor $(\mathrm{Q})$; deducted in this study, which is less than the stipulated for ductile structures. In order to illustrate this two reinforced concrete frames have been designed: one for $\mathrm{Q}=4$ and the other one for the maximum value of $\mathrm{Q}$. It is shown that the frame designed for $\mathrm{Q}$ $=4$ responds inelastically when acting the design earthquake associated with the serviceability limit state, and therefore it does not meet the required performance parameters. Furthermore, it is shown that the frame designed for the maximum value of $\mathrm{Q}$, responds elastically when acting the design
\end{abstract}

Artículo recibido el 29 de julio de 2014 y aprobado para su publicación el 8 de mayo de 2015. Se aceptarán comentarios y/o discusiones hasta cinco meses después de su publicación.

(1) Profesor, Facultad de Ingeniería Civil, Universidad Veracruzana. Circuito G. Aguirre Beltrán. Col. Lomas del estadio, C.P.: 91090, Xalapa, Veracruz, e-mail: erbarradas@uv.mx

(2) Profesor investigador, Instituto de Ingeniería, UNAM, Coordinación de Mecánica aplicada. Circuito Escolar, Ciudad Universitaria, Coyoacán, 04510, México D.F, e-mail: GAyalaM@iingen.unam.mx 
earthquake associated with the serviceability limit state, and therefore meets the established performance parameters.

Keywords: Over-strength factor; Seismic behavior factor; Multilevel seismic design of buildings

\section{INTRODUCCIÓN}

En el apéndice A (AA), de las Normas Técnicas Complementarias para Diseño por Sismo del 2004 (NTCS-2004), se estipula un procedimiento de análisis y diseño sísmico multinivel (diseño para dos o más estados límite), en el que se establece el cumplimiento de dos estados límite: de servicio (ELS) y de falla o de prevención del colapso (ELPC). Se define además el espectro de diseño correspondiente a cada estado límite. De acuerdo a Ordaz et al., (2003), estos espectros tienen formas espectrales e intensidades realistas, y para efectuar las reducciones de resistencia por ductilidad, se emplean reglas más acordes con las reducciones que se observan en los diferentes sitios del valle de México para sistemas de un grado de libertad. Cabe mencionar; sin embargo, que Ordaz et al. (2003) reconocen que los valores propuestos para la sobrerresistencia estructural $(R)$ y la capacidad dúctil global de una estructura, (factor de comportamiento sísmico $(Q)$ en las NTCS-2004), no están plenamente justificados con bases teóricas o empíricas. Esta situación es común en la mayoría de los reglamentos de diseño sísmico internacionales.

Por otra parte, siempre que el proyecto arquitectónico lo permita, los ingenieros adecuan los sistemas estructurales para que cumplan con ciertos requisitos reglamentarios de regularidad en cuanto a su configuración geométrica y distribución de masas, rigideces y resistencias; y resulten en estructuras "regulares", ya que éstas exhiben un mejor comportamiento sísmico. Además, si en los miembros de estas estructuras y en las conexiones entre ellos se cumplen con ciertos requisitos geométricos y de detallado sísmico, los reglamentos suelen clasificarlas como "estructuras dúctiles" y permiten diseñarlas para los máximos valores de $Q$. Esto hace posible poder reducir al máximo las fuerzas sísmicas de diseño, con lo que se espera obtener diseños significativamente más económicos. Es evidente; sin embargo, que el detallado sísmico que requiere una estructura dúctil incrementa su tiempo de construcción, el costo de la mano de obra y de la supervisión de la misma.

En este trabajo se muestra que a pesar de que las estructuras que se ubicarán sobre suelos del valle de México con periodos dominantes (Ts) mayores a $1.65 \mathrm{~s}$ y diseñadas con lineamientos del AA de las NTCS-2004, satisfagan los requisitos establecidos por dichas normas para el uso de los valores de $Q$ correspondientes a estructuras dúctiles $(Q=3$ y $Q=4)$, para que dichas estructuras cumplan los parámetros de desempeño establecidos en el AA, deben diseñarse para un valor máximo de $Q$; deducido en el presente estudio, que es menor a los estipulados para estructuras dúctiles. Para ilustrar lo anterior, se diseñan dos marcos de concreto reforzado: uno para un $Q=4$ y otro para el valor máximo de $Q$. Se demuestra que el marco diseñado para $Q=4$ responde inelásticamente al sismo de diseño asociado al ELS, y por tanto que no cumple los parámetros de desempeño requeridos. También se demuestra que el marco diseñado para el valor máximo de $Q$, al responder elásticamente al sismo de diseño asociado al ELS, cumple los parámetros de desempeño establecidos.

\section{CRITERIOS DE DESEMPEÑO ESTABLECIDOS EN EL APÉNDICE A DE LAS NTCS-2004}

En el AA de las NTCS-2004 se estipula que las estructuras deben diseñarse para satisfacer simultáneamente los siguientes criterios de desempeño (Ordaz et al. 2003): 
I. Resistir sismos de baja a moderada intensidad respondiendo elásticamente y presentando un daño no estructural incipiente.

II. No colapsar ante los sismos de mayor intensidad que puedan ocurrir en el sitio, aunque se presente cierto daño estructural y no estructural.

Para satisfacer el primer estado de daño de la estructura, al cual se le denomina ELS, las NTCS2004 solo indican que se limite la máxima distorsión de entrepiso a un valor para el cual se inicia el daño en elementos no estructurales. El segundo estado de daño, denominado ELPC, se considera satisfecho si se suministra a la estructura: (1) una resistencia lateral tal que su demanda global de ductilidad ante el sismo de diseño, sea menor o igual a la capacidad dúctil global de la estructura, y (2) una rigidez lateral suficiente para que ante el sismo de diseño, la máxima distorsión de entrepiso sea menor o igual a la permisible para éste estado, la cual define la capacidad de desplazamiento relativo de entrepiso del sistema estructural.

La fig. 1, adaptada de Uang, (1991), muestra para una estructura capaz de disipar energía de manera estable, sus curvas de respuesta global; real e idealizada, definidas por la relación entre su coeficiente sísmico $(C)$ y su distorsión de entrepiso $(4) . C_{e}$ es el coeficiente sísmico elástico, el cual se define mediante la ec. 1:

$C_{e}=\frac{V_{b e}}{W}$

Donde $V_{b e}$ es el cortante basal requerido para que la estructura permanezca elástica ante el sismo de diseño asociado al ELPC, y $W$ es el peso de la estructura.

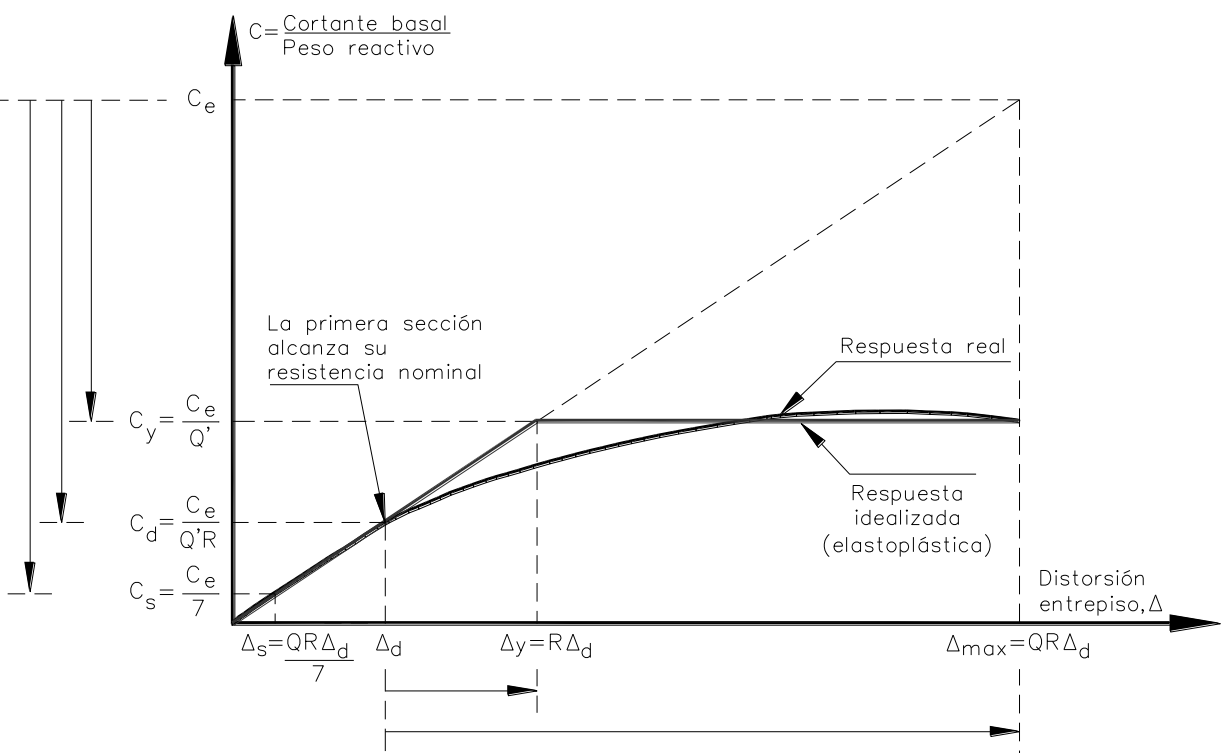

Figura 1. Relación coeficiente sísmico basal-máxima distorsión de entrepiso de una estructura

$C d$ es el coeficiente sísmico de diseño y se define mediante la ec. 2 :

$C_{d}=\frac{C_{e}}{Q^{\prime} R}$ 
donde $Q^{\prime}$ es el factor de reducción de fuerzas sísmicas por ductilidad. De acuerdo a Uang, 1991, el nivel de fuerza definido por $C_{d}$ es consistente con el proporcionado a los miembros por medio de los procedimientos simplificados indicados en los reglamentos para el cálculo de sus resistencias de diseño. Este nivel de fuerza define el límite del comportamiento elástico de la respuesta global de la estructura. La distorsión de entrepiso correspondiente a $C_{d}$ es $\Delta_{d}$.

$C_{y}$ es el coeficiente sísmico correspondiente a la resistencia de la estructura para la cual, de manera idealizada, se inicia el desarrollo de su mecanismo de colapso; y se define mediante la ec. 3:

$C_{y}=\frac{C_{e}}{Q^{\prime}}$

$Q$ ' es función de $Q$, del periodo de vibración estructural $(T)$ y de $T s$.

La distorsión de entrepiso correspondiente a $C_{y}$ es $\Delta_{y}$, y se define mediante la ec. 4. Esta distorsión es la correspondiente al inicio del desarrollo del mecanismo de colapso, y por tanto se presenta dentro del intervalo de comportamiento inelástico de la estructura.

$\Delta_{y}=R \Delta_{d}$

La distorsión de entrepiso inelástica máxima de la estructura $\left(\Delta_{\max }\right)$, se define mediante la ec. 5.

$\Delta_{\max }=Q \Delta_{y}=Q R \Delta_{d}$

$C_{s}$ es el coeficiente sísmico correspondiente al ELS y se define mediante la ec. 6:

$C_{s}=\frac{C_{e}}{7}$

donde la constante siete, por las razones que se discutirán en la siguiente sección, es la cantidad por la que se debe dividir el cortante basal elástico de diseño de la estructura para obtener el correspondiente al ELS.

La distorsión de entrepiso correspondiente a $C_{s}$ es $\Delta_{s}$, y se puede obtener mediante la ec. 7:

$\Delta_{s}=\frac{Q R \Delta_{d}}{7}$

Cabe mencionar que en la ecuación anterior implícitamente se considera que $\Delta_{\max }$ es igual a la distorsión máxima del sistema elástico-lineal correspondiente $\left(\Delta_{e}\right)$, lo cual implica que es válida la aplicación de la regla de iguales desplazamientos. Como es conocido, la aplicación de esta regla es razonable solo en las regiones espectrales de periodos intermedios y largos. 


\section{DESCRIPCIÓN DEL PROBLEMA}

Para que una estructura con una $R$ dada responda elásticamente al sismo de diseño correspondiente al ELS se debe tener que $C_{d} \geq C_{s}$, fig. 1. Para cumplir esta igualdad, es evidente de las ecs. 2 y 6 que el valor máximo del $Q$ de diseño debe ser tal que: $Q^{\prime}(Q) * R \leq 7$.

En la fig. 2 se comparan los espectros de diseño en algunos sitios del valle de México estipulados en el AA de las NTCS-2004 para el ELPC, reducidos por distintos valores del producto $Q$ ' $R$; siendo los $Q^{\prime}$ correspondientes a: $Q^{\prime}(Q=2), Q^{\prime}(Q=3)$ y $Q^{\prime}(Q=4)$, con los espectros elásticos de diseño correspondientes al ELS. Es importante mencionar que el espectro de diseño para el ELS no está explícitamente definido, sin embargo, la revisión de los desplazamientos laterales que requieren dichas normas para este estado se debe hacer con los desplazamientos obtenidos del espectro elástico de diseño correspondiente al ELPC divididos entre siete, lo que implica que de manera indirecta se considera que el espectro de servicio tiene la misma forma que el de diseño para el ELPC y sus ordenadas espectrales son siete veces menores.

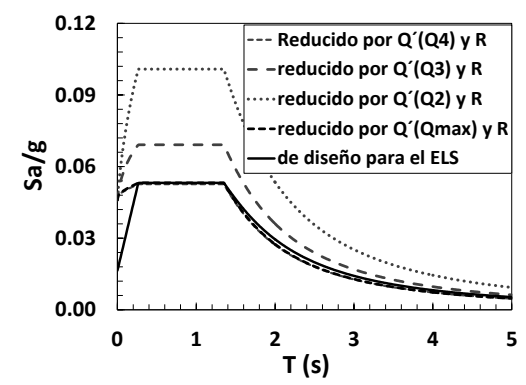

a). Sitio con $T s=0.6 \mathrm{~s}$

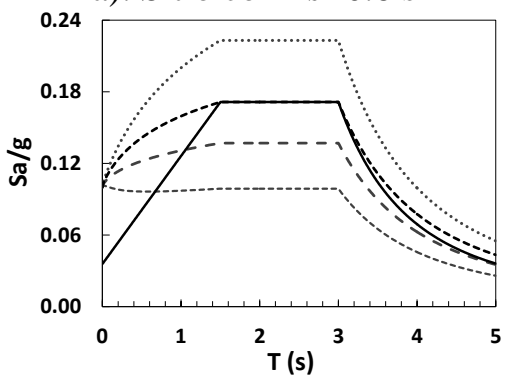

d). Sitio con $T s=2.5 \mathrm{~s}$

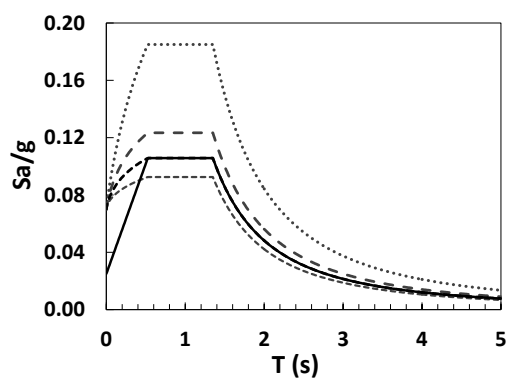

b). Sitio con $T s=1.0 \mathrm{~s}$

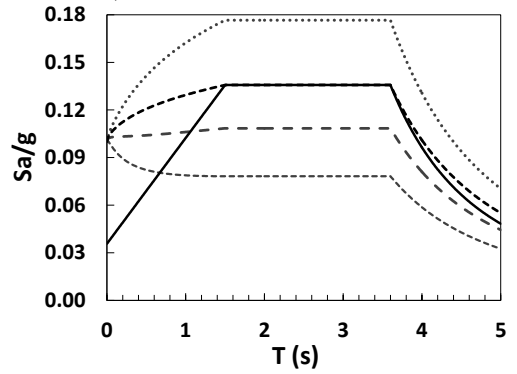

e). Sitio con $T s=3.0 \mathrm{~s}$

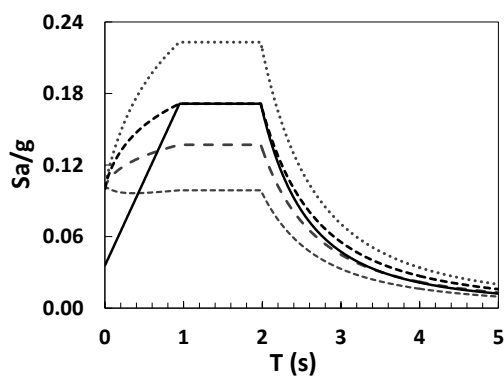

c). Sitio con $T s=1.65 \mathrm{~s}$

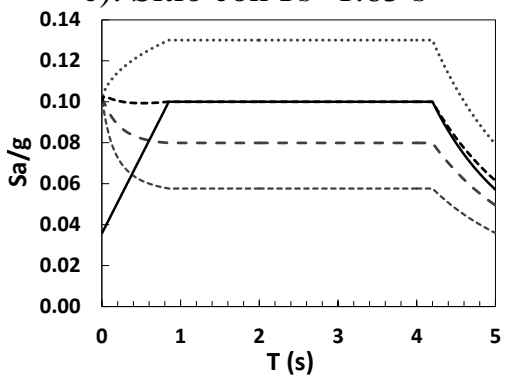

f). Sitio con $T s=4.0 \mathrm{~s}$

Figura 2. Comparación de los espectros de diseño en algunos sitios del valle de México estipulados en el AA de las NTCS-2004, para el ELPC; reducidos por distintos valores del producto $Q^{\prime} R$, y los estipulados para ELS.

En todos los sitios se observa que, a excepción de una parte de la rama ascendente de los espectros (región de periodos cortos), las ordenadas de los espectros inelásticos de diseño correspondientes a $Q=4$ son de ligera a considerablemente menores a las de los espectros de diseño asociados al ELS. Se puede observar una situación similar con los espectros de diseño correspondientes a $Q=3$ en sitios cuyo Ts es igual o mayor a $1.65 \mathrm{~s}$. De las observaciones anteriores, y teniendo en cuenta que el cortante basal de diseño de una estructura se calcula a partir del espectro inelástico o reducido, se puede inferir que una estructura diseñada para $Q=4$ o $Q=3$, para cuyo periodo fundamental la correspondiente ordenada en el espectro inelástico sea menor a la respectiva ordenada del espectro correspondiente al ELS, responderá inelásticamente al sismo de diseño asociado al ELS. Más aún, dado que en esta condición la estructura experimentará cierta pérdida de su rigidez lateral, sus máximas demandas de deformación lateral serán 
mayores a las estimadas en el diseño para el ELS, por lo que los elementos no estructurales presentarán cierto nivel de daño.

Algunas implicaciones de no cumplir los parámetros de desempeño requeridos para el ELS son: (1) de acuerdo a Ordaz et al. (2003), las ordenadas del espectro de diseño para el ELS son similares a las de espectros de respuesta de sismos que tienen un periodo de retorno de alrededor de diez años. Si las ordenadas del espectro reducido por $Q^{\prime}$ y $R$ son menores a las del espectro correspondiente al ELS, las ordenadas del primer espectro estarían asociadas a un periodo de retorno menor a diez años, por lo que las estructuras diseñadas con este espectro exhibirán cierto nivel de daño estructural y no estructural ante sismos con mayor frecuencia de ocurrencia que el de servicio. (2) Dependiendo de la magnitud del daño que exhiban los miembros estructurales, los costos de rehabilitación, sumados a los de interrupción de labores por estas actividades pueden ser considerables; sin embargo, las mayores pérdidas económicas posiblemente se deberán al daño en elementos no estructurales; cuyos costos, dependiendo del tipo y área total de estos elementos, pueden representar del 12\% al 33\% del costo total de la edificación (Mayes, 1995).

\section{ALTERNATIVAS PARA SOLUCIONAR EL PROBLEMA}

El problema discutido muestra que si se desea que una estructura responda elásticamente al sismo de diseño asociado al ELS, se debe diseñar para un valor de $Q$ tal que; en el intervalo de periodos que comprenda la estructura, las ordenadas del espectro reducido correspondiente sean mayores, o cuando menos iguales a las ordenadas del espectro de diseño asociado al ELS. En el caso de que las ordenadas de ambos espectros sean iguales, el valor de $Q$ definirá el máximo para el que se deba diseñar la estructura. En la fig. 2 se muestra que para un valor de $Q=2$, en todos los sitios y para un intervalo de periodos de cero a más de $5 \mathrm{~s}$, las ordenadas de los espectros de diseño reducidos por $Q^{\prime}\left(Q^{\prime}=2\right)$ y $R$ son mayores a las correspondientes del espectro de diseño asociado al ELS, por lo que al usar este valor de $Q$, se lograría que la estructura responda elásticamente al sismo de diseño correspondiente al ELS.

\section{Obtención de un valor máximo de diseño del factor de comportamiento sísmico}

Si por razones económicas se desea reducir las fuerzas sísmicas elásticas de diseño al máximo, pero logrando que la estructura cumpla los criterios de desempeño establecidos para el ELS, se debe diseñar para cierto valor máximo de $Q$. Debido a que las ordenadas de los espectros inelásticos correspondientes a valores de $Q=3$ y $Q=4$ son menores a las del espectro de servicio en prácticamente el intervalo completo de periodos que presentan estructuras con características comunes, se propone obtener valores máximos de $Q$ aplicables a sitios. De acuerdo con lo anterior, el valor máximo de $Q$ se debe obtener en la región espectral en la que las reducciones de resistencia por ductilidad sean máximas. En la fig. 3, tomada de Ordaz et al. (2003), se muestran las gráficas de la función $Q^{\prime}(Q=4)$ correspondientes a diversos valores de $T s$. Se observa, entre diferentes aspectos, que los máximos valores de $Q$ ' en la mayoría de los suelos se presentan en la meseta de los espectros de diseño. Considerando este hecho, se propone calcular un valor máximo del factor de comportamiento sísmico $\left(Q_{\max }\right)$, tal que las ordenadas de la meseta del espectro inelástico correspondiente a $Q^{\prime}\left(Q_{\max }\right)$ y al valor de $R$ indicado en las NTCS-2004, sean iguales a las correspondientes del espectro de diseño asociado al ELS. El valor de $Q_{\max }$ será el máximo para el que deban diseñarse las estructuras a ubicarse en el sitio en cuestión, de tal forma que respondan elásticamente al sismo de diseño asociado al ELS. 


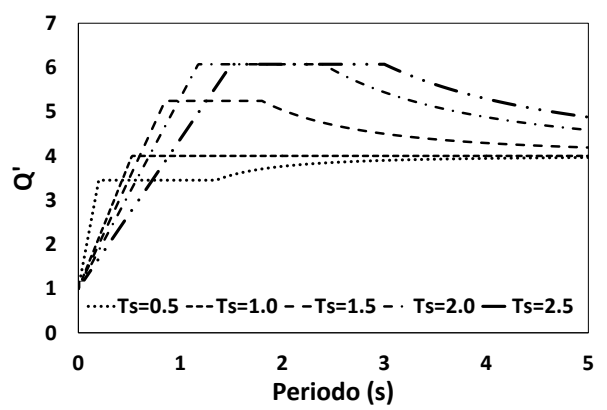

Figura 3. Gráfica de la función $Q^{\prime}(Q, T, T s)$ para $Q=4$ y los valores de $T s$ mostrados

En la ecuación A.9 del AA de las NTCS-2004, $Q^{\prime}$ en la meseta de los espectros de diseño se define como:

$Q^{\prime}=1+(Q-1) \sqrt{\frac{\beta}{k}}$

donde $\beta$ es un factor de reducción por amortiguamiento suplementario, que es igual a uno cuando se ignora la interacción suelo-estructura, y $k$ es una constante, que de acuerdo a la ecuación A.6 del AA de las NTCS-2004, presenta los valores:

$k=\left\{\begin{array}{l}2-T s ; \text { si } 0.5<T s \leq 1.65 s \\ 0.35 \text { si Ts }>1.65 s\end{array}\right.$

El valor requerido de $Q_{\max }$ para que las ordenadas en la meseta de ambos espectros de diseño tengan el mismo valor, se puede determinar a partir de la siguiente ecuación, en la que su primer término representa la ordenada en el espectro correspondiente al ELS y su segundo término la ordenada en el espectro correspondiente al ELPC.

$\frac{S a}{7}=\frac{S a}{C I\left[1+(Q-1) \sqrt{\frac{\beta}{k}}\right] R}$

donde $S a$ es la ordenada de seudoaceleración en la meseta del espectro elástico de diseño asociado al ELPC, $C I$ es la corrección que se aplica al factor de reducción de fuerzas sísmicas debida a la irregularidad de la estructura, y la ecuación entre corchetes en el denominador del segundo término es el valor de $Q^{\prime}$ definido en la ec. 8.

Después de realizar algunas operaciones algebraicas sobre la ec. 10 se obtienen las siguientes ecuaciones para $Q_{\max }$ :

Para suelos con $0.5<T s \leq 1.65 \mathrm{~s}$ :

$Q_{\text {max }}=\frac{(7-C I R) \sqrt{2-T s}+C I R \sqrt{\beta}}{C I R \sqrt{\beta}}$

Para suelos con $T s>1.65 \mathrm{~s}$ : 
$\mathrm{Q}_{\max }=\frac{(7-C I R) \sqrt{0.35}+C I R \sqrt{\beta}}{C I R \sqrt{\beta}}$

$Q_{\max }$ se puede interpretar como la demanda de ductilidad global correspondiente a la resistencia lateral requerida por el sismo de diseño asociado al ELS.

Es importante notar que el problema discutido no es consecuencia de la forma y amplitud de la función $Q^{\prime}(Q, T, T s)$. El problema es que debido a la magnitud de las ordenadas que tienen los espectros de diseño correspondientes al ELS en estos sitios, la resistencia lateral requerida en el ELS rige la máxima demanda de ductilidad global, $Q_{\max }$, que debe desarrollar la estructura, la cual es menor a la capacidad dúctil prescrita, $Q$, para estructuras dúctiles. Cabe mencionar además, que si el valor requerido de $Q_{\max }$ es menor a los que estipulan las Normas Técnicas Complementarias para Diseño y Construcción de Estructuras de Concreto del 2004 (NTCC-2004), para estructuras dúctiles ( $Q=3$ y $Q=4$ ), es recomendable (Rodríguez y Restrepo, 2012), se apliquen los requisitos establecidos por dichas normas para el diseño y construcción de estructuras dúctiles. Esto se debe a que, como se conoce, existen grandes incertidumbres en la estimación de las acciones sísmicas de diseño, y por tanto, no es posible garantizar que las estructuras no se sujetarán a demandas de deformación mayores a las estimadas en el diseño.

Como ejemplo de aplicación de las ecs. 11 y 12, de acuerdo a la ecuación A.10 del AA de las NTCS-2004, en la meseta de los espectros de diseño el valor de $R$ es igual a 2. Si además se considera que $C I$ es igual a la unidad y se ignora la interacción suelo-estructura, se tiene que para suelos con $T s>1.65 \mathrm{~s}$, $Q_{\max }=2.48$, y para un suelo con $T s=0.6 \mathrm{~s}, Q_{\max }=3.96$. En la fig. 2 se muestran los espectros inelásticos de diseño correspondientes a $Q^{\prime}\left(Q_{\max }\right)$ y $R$, para los sitios del valle de México mencionados anteriormente.

\section{EJEMPLOS ILUSTRATIVOS}

Con la finalidad de mostrar las implicaciones de definir el cortante basal de diseño $\left(V b_{D}\right)$ de una estructura con un valor menor, y otro aproximadamente igual al correspondiente del ELS ( $\left.V b_{E L S}\right)$, sobre el desempeño esperado de la estructura y sus elementos no estructurales en dicho estado límite, se diseñó un marco interior de la estructura mostrada en la fig. 4 para ambos valores de cortante basal. Posteriormente se determinaron las propiedades mecánicas globales de rigidez y resistencia de estos marcos, a partir de su curva de respuesta cortante basal-deformación lateral máxima obtenida de un análisis estático inelástico ante un patrón de fuerzas laterales monótonamente crecientes. Finalmente, en esta curva de respuesta global se identificó el estado de daño y la magnitud de las deformaciones laterales máximas de la estructura cuando se desarrolla el $V b_{E L S}$.

\section{Ejemplo 1. Estructura cuyo $V b_{D}$ es menor $V b_{E L S}$, (marco E1)}

Se diseñó el marco interior ubicado en el eje $\mathrm{C}$, que forma parte del edifico a base de marcos rígidos de concreto reforzado, cuyas configuraciones en planta y elevación se muestran en la fig. 4 . El sistema de piso del edificio es a base de losas macizas de concreto reforzado de $12 \mathrm{~cm}$ de espesor, coladas monolíticamente con las vigas. La edificación se ubica en un sitio con un $T s=2.0 \mathrm{~s}$, para el cual, de acuerdo a la ecuación A.3 del apéndice A, el coeficiente sísmico tiene un valor de 1.2. El uso del edificio es para oficinas y las cargas gravitacionales consideradas se muestran en la tabla 1. Para el diseño se eligió un $Q=4$, y se determinó que no es necesario aplicar un $C I$ menor a la unidad. En la fig. 5 se muestra el espectro inelástico o reducido por $Q^{\prime}(Q=4)$ y $R$, así como el espectro elástico de diseño correspondiente al ELS. Ambos espectros se calcularon para una fracción de amortiguamiento respecto al crítico del $5 \%$. Las 
propiedades mecánicas de los materiales usadas para el análisis y diseño de la estructura son, para el concreto: concreto tipo I con $f^{\prime} c=250 \mathrm{kgf} / \mathrm{cm}^{2}$ y $E=221360 \mathrm{kgf} / \mathrm{cm}^{2}$, y para el acero: $f y=4200 \mathrm{kgf} / \mathrm{cm}^{2}$.

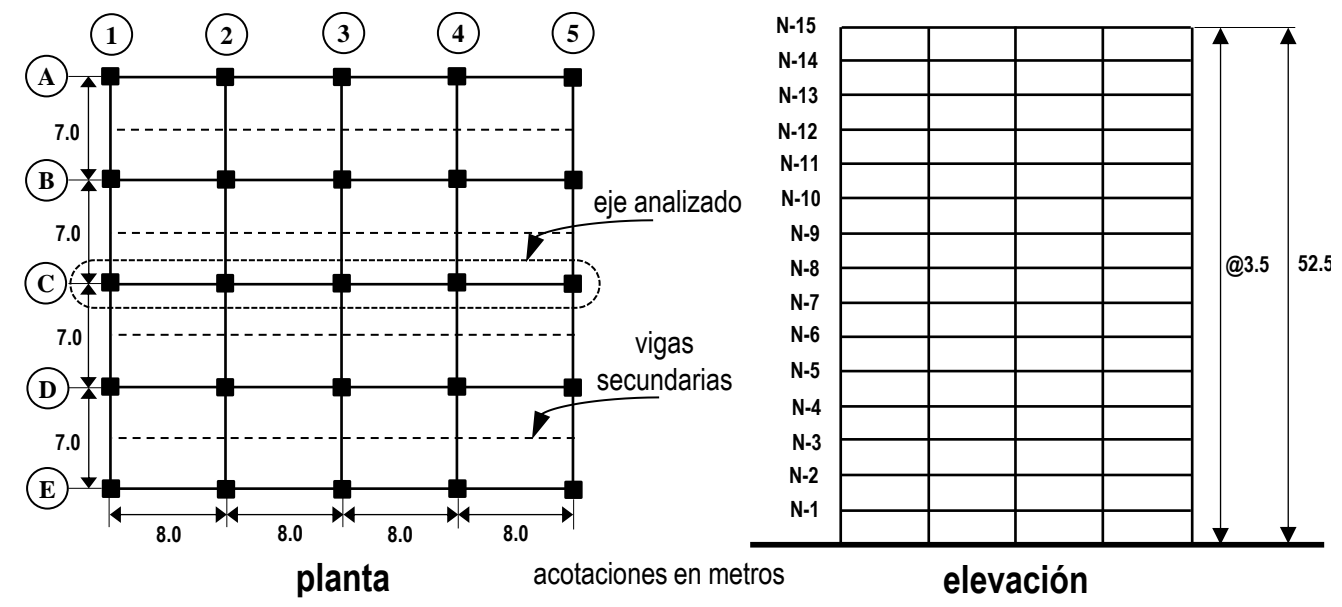

Figura 4. Configuraciones en planta y elevación del edificio que contiene al marco estudiado

Tabla 1. Cargas gravitacionales consideradas

\begin{tabular}{rc}
\hline Cargas gravitacionales: & $\left(\mathrm{kgf} / \mathrm{m}^{2}\right)$ \\
\hline Carga muerta en azotea $=$ & 435 \\
Carga viva instantánea en azotea $=$ & 70 \\
Carga viva máxima en azotea $=$ & 100 \\
Carga muerta en entrepiso $=$ & 480 \\
Carga viva instantánea en entrepiso $=$ & 180 \\
Carga viva máxima en entrepiso $=$ & 250 \\
\hline
\end{tabular}

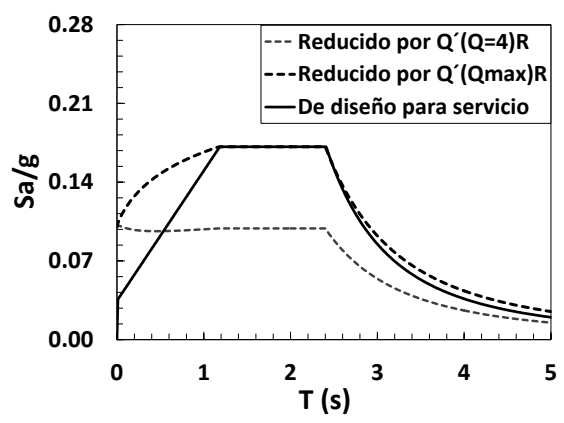

Figura 5. Acciones sísmicas correspondientes a los estados límite de diseño

Para el análisis estructural del marco se consideró que la sección transversal de las vigas es rectangular en toda su longitud, por lo que no se consideró la contribución de la losa ni a la resistencia ni a la rigidez de las vigas. Para tomar en cuenta la pérdida de rigidez debida al agrietamiento en los miembros estructurales, se consideraron los valores de los momentos de inercia efectivos indicados por las NTCC2004. También se consideraron los efectos de segundo orden, y se ignoró la interacción suelo-estructura. Los miembros estructurales se diseñaron y detallaron de acuerdo a los lineamientos del capítulo 7 de las NTCC-2004. Para el cálculo de las resistencias de diseño en las secciones transversales de las vigas, no se consideró la contribución del refuerzo a compresión. Las distorsiones de entrepiso permisibles consideradas son: $0.4 \%$ para el ELS y $3 \%$ para el ELPC. Los análisis estructurales elásticos realizados al marco consisten en análisis modales espectrales llevados a cabo con el programa SAP 2000 v.14 (CSI, 
2010). En dichos análisis se consideró que todos los modos tienen una fracción de amortiguamiento respecto al crítico del $5 \%$.

El valor requerido para el periodo fundamental del marco E1 es de $1.65 \mathrm{~s}$, el cual se ubica en la meseta de los espectros de diseño. El peso total del marco es de 2049 ton. La revisión por cortante basal se realizó verificando que el $V b_{D}=166.32$ ton, no fuera menor que el valor dado por $0.8 a \mathrm{Wo} / Q^{\prime} R$. Puesto que se ignoró la interacción suelo-estructura, $\beta=1$, por lo tanto, de la ecuación A.1 del AA: $a=c=1.2$. Debido a que el periodo fundamental se ubica en la meseta del espectro, $R$ tiene un valor de 2 . Puesto que $T s=2.0 \mathrm{~s}$ $>1.65 \mathrm{~s}, k=0.35$, por lo tanto, $Q^{\prime}=6.071$. Con estos datos se puede verificar que: 166.32 ton > $(0.8 * 1.2 * 2049) /(6.071 * 2)=162$ ton. En la tabla 2 se muestran las dimensiones requeridas para las secciones transversales de los miembros estructurales, así como las cuantías de refuerzo longitudinal necesarias para suministrarles las resistencias de diseño a momento negativo $(\rho$-) y a momento positivo $(\rho+)$ en sus secciones extremas. El hecho de que las cuantías requeridas sean bajas indica que el diseño fue regido por rigidez y no por resistencia. En la fig. 6 se muestran los armados de las secciones transversales extremas de los miembros estructurales. Aunque no se muestran las cuantías refuerzo longitudinal requeridas en las secciones transversales intermedias de las vigas, dichas cuantías cumplen con el requisito que establece que en ninguna sección a lo largo de la viga, ni el momento resistente negativo, ni el resistente positivo, serán menores que la cuarta parte del máximo momento resistente que tenga la viga en sus secciones extremas. El refuerzo longitudinal de las losas consiste en varillas del no.3, colocadas a cada $15 \mathrm{~cm}$ en el lecho superior, y el refuerzo mínimo requerido en el lecho inferior.

Tabla 2. Dimensiones y cuantías de refuerzo requeridas para las secciones transversales extremas de los miembros estructurales de los marcos estudiados.

\begin{tabular}{ccccc}
\hline & & Dimensiones(m) & $\rho l(\%)$ & $\rho l(\%)$ \\
& Nivel & Marcos E1 y E2 & Marco E1 & Marco E2 \\
\hline \multirow{3}{*}{ Columnas } & 1 a 5 & $1.10 \times 1.10$ & 1.00 & 1.32 \\
& 6 a 10 & $0.95 \times 0.95$ & 1.06 & 1.06 \\
& 11 a 15 & $0.80 \times 0.80$ & 1.11 & 1.11 \\
\hline \multirow{3}{*}{ Vigas } & 1 a 8 & $0.40 \times 1.00$ & $\rho l^{-}=0.76, \rho l^{+}=0.42$ & $\rho l=1.16, \rho l^{+}=0.82$ \\
& 9 a 12 & $0.40 \times 0.85$ & $\rho l=0.83, \rho l^{+}=0.41$ & $\rho l=1.06, \rho l^{+}=0.63$ \\
& 13 a 15 & $0.40 \times 0.85$ & $\rho l^{\circ}=0.50, \rho l^{+}=0.41$ & $\rho l=0.63, \rho l^{+}=0.41$ \\
\hline
\end{tabular}

En la fig. 7 se muestran los desplazamientos laterales de los niveles y las distorsiones de entrepiso para los dos estados límite de diseño. Se muestran además, los desplazamientos laterales de los niveles y las distorsiones de entrepiso correspondientes al $V b_{D}=166.32$ ton, a los cuales se les denomina como desplazamientos y distorsiones de "diseño". Se puede apreciar que las deformaciones laterales de diseño son menores a las correspondientes del ELS, lo cual era de esperarse debido a que el valor de la ordenada espectral correspondiente al periodo fundamental en el espectro reducido por $Q^{\prime}$ y $R$, es menor a la correspondiente ordenada en el espectro de diseño correspondiente al ELS. Este resultado indica, desde la etapa del diseño, que solo algunos de los miembros de la estructura desarrollan un comportamiento inelástico, y por tanto, la estructura exhibirá solo un comportamiento global inelástico incipiente antes de alcanzar el $V b_{E L S}$. 


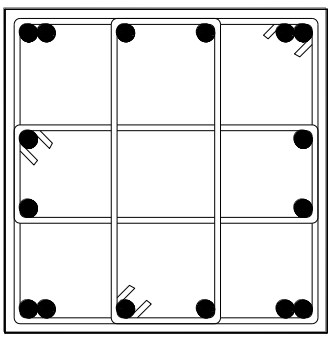

columnas niveles 1 a 5 16 no. 10

30no.4: 11@10, resto@40c/e

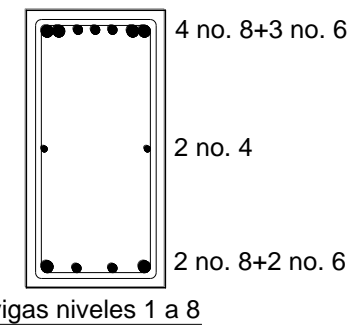
vigas niveles 1 a 8

10no.3:1@5,15@13,resto@16c/e

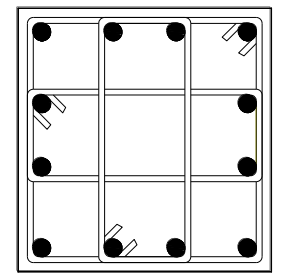

columnas niveles 6 a 10 12 no. 10

30no.4: 10@10, resto@40c/e

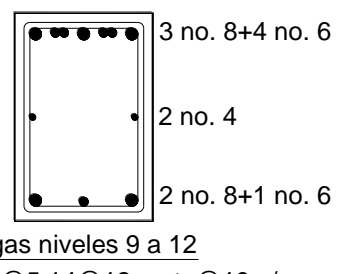

10no.3:1@5,14@12,resto@19c/e

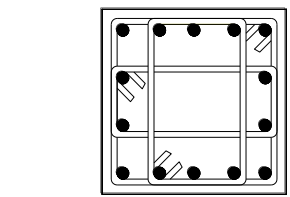

columnas niveles 11 a 15 14 no. 8 3งno. 4: 8@10, resto@30c/e

Nota: c/e significa al centro del elemento

Figura 6. Detalle del refuerzo requerido en las columnas y vigas del marco E1

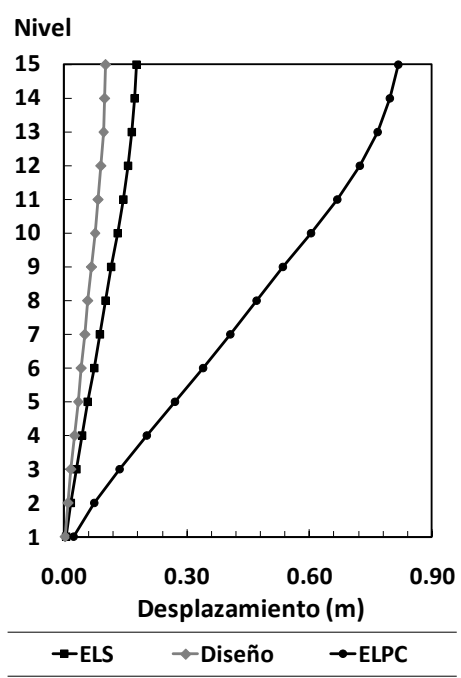

a). Desplazamientos de los niveles

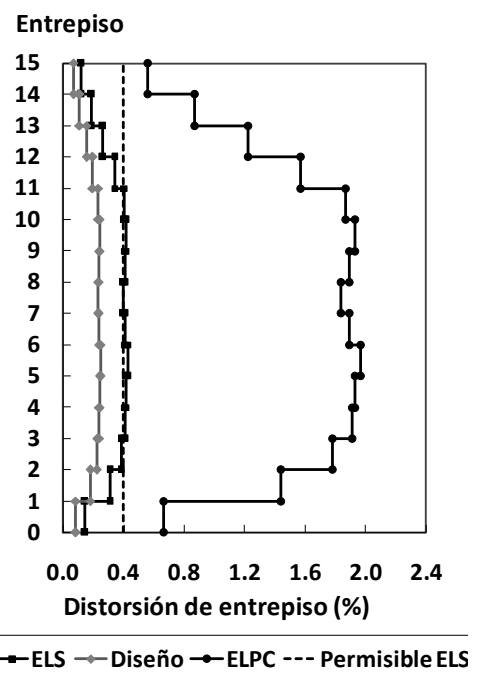

b). Distorsiones de entrepiso

Figura 7. Deformaciones laterales de diseño del marco E1

\section{Ejemplo 2. Estructura cuyo $V b_{D}$ es similar al $V b_{E L S}$, (marco E2)}

En este ejemplo se diseña el mismo marco del ejemplo E1, pero considerando como acción sísmica de diseño asociada al ELPC el espectro reducido por $Q_{\max }=2.479$ y $R$, el cual se muestra en la fig. 5 . 
El valor requerido para el periodo fundamental del marco E2 es el mismo que el del marco E1, 1.65 s, lo cual se debe a que el diseño del marco E1 fue "regido" por el ELS. El peso total del marco es de 2049 ton. La revisión por cortante basal se realizó verificando que el $V b_{D}=287.4$ ton, no fuera menor que el valor dado por $0.8 a W o / Q^{\prime} R$. De manera similar al ejemplo anterior: $\beta=1, a=c=1.2, R=2$ y $k=0.35$, por lo tanto, $Q^{\prime}(Q=2.479)=3.5$. Con estos datos se verifica que: 287.4 ton $>\left(0.8^{*} 1.2 * 2049\right) /(3.5 * 2)=281.0$ ton. En la tabla 2 se muestran las dimensiones requeridas para las secciones transversales de los miembros estructurales, así como las cuantías de refuerzo necesarias para suministrarles las resistencias de diseño. La fig. 8 muestra el detalle de los armados de las secciones transversales de los miembros estructurales.

En la fig. 9 se muestran los desplazamientos laterales de los niveles y las distorsiones de entrepiso correspondientes a los dos estados límite de diseño, así como las correspondientes al $V b_{D}=287.4$ ton. Se puede apreciar que tanto el $V b_{D}$ como las correspondientes deformaciones laterales de diseño, son ligeramente mayores a las correspondientes del ELS, debiéndose dicha diferencia a la mayor contribución de los modos superiores en el espectro reducido por $Q^{\prime}\left(Q_{\max }\right)$ y $R$, como se aprecia en la fig. 5. Este resultado indica, desde la etapa del diseño, que la estructura incursiona en su intervalo de comportamiento inelástico cuando exceda casi simultáneamente los $V b_{D}$ y $V b_{E L S}$.

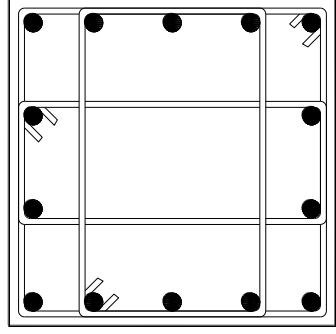

columnas niveles 1 a 5 14 no. 12

3৩no.4: 11@10, resto@40c/e

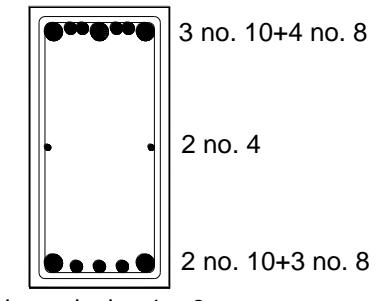

vigas niveles 1 a 8

10no.3:1@5,22@9,resto@10c/e

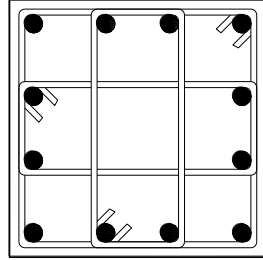

columnas niveles 6 a 10

$$
12 \text { no. } 10
$$

3৩no.4:10@10, resto@40c/e

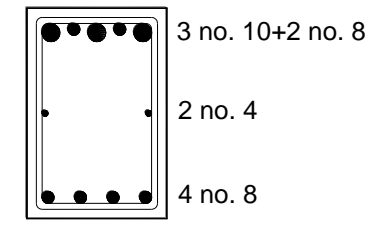

vigas niveles 9 a 12 10no.3:1@5,15@11resto@14c/e

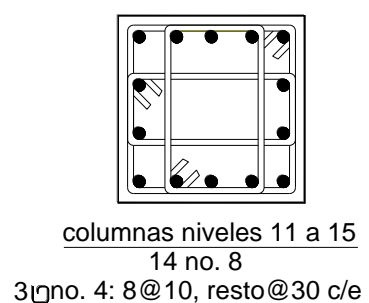

3৩no.4:8@10, resto@30c/e

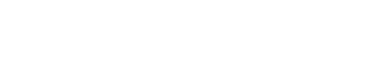

Nota: c/e significa al centro del elemento

Figura 8. Detalle del refuerzo requerido en las columnas y vigas del marco E2 


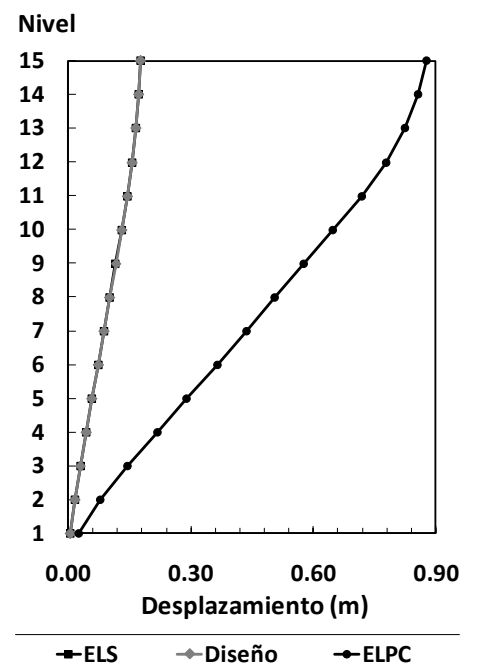

a). Desplazamientos de los niveles

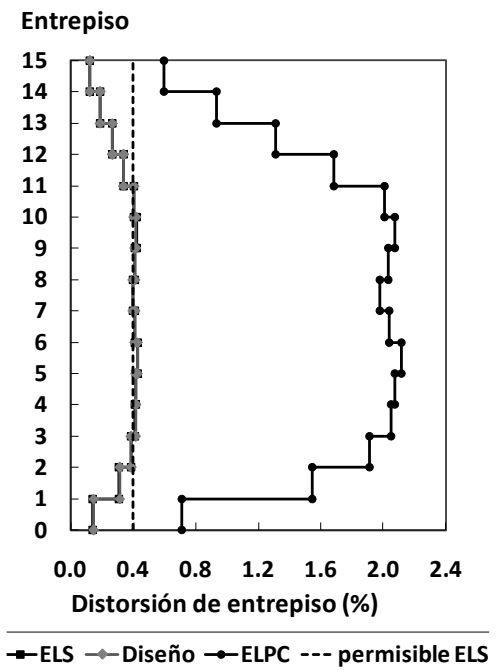

b). Distorsiones de entrepiso

Figura 9. Deformaciones laterales de diseño del marco E2

\section{Propiedades mecánicas globales de las estructuras}

Una forma de obtener una aproximación de las propiedades mecánicas globales de rigidez, resistencia y capacidad de deformación laterales de una estructura, es a partir de su curva de respuesta inelástica cortante basal-deformación lateral máxima. Un procedimiento comúnmente empleado para calcular dicha curva, es el denominado análisis pushover, el cual es un análisis inelástico que consiste en aplicar estáticamente en los niveles o pisos de una estructura, un patrón de fuerzas laterales monótonamente crecientes, hasta que algunos de los elementos estructurales alcanzan su capacidad rotacional última.

\section{Propiedades mecánicas de los elementos estructurales}

La resistencia, rigidez y capacidad de deformación de los elementos estructurales se obtuvieron a partir de una idealización bilineal de los diagramas momento-curvatura de sus secciones transversales extremas. Para el cálculo de estos diagramas se hicieron las siguientes consideraciones: (1) Las relaciones esfuerzo-deformación para el concreto confinado (concreto del núcleo de las secciones) y no confinado (concreto del recubrimiento) se definieron mediante el modelo propuesto por Mander et al., (2000). (2) La relación esfuerzo-deformación del acero de refuerzo se definió mediante el modelo sugerido por Rodríguez y Botero (1996). Los valores de los esfuerzos y deformaciones unitarias del acero que definen los diferentes puntos característicos de su curva esfuerzo-deformación, se tomaron como los valores medios de dichas propiedades sugeridos por los mismos autores. (3) La carga axial considerada para el cálculo de los diagramas momento-curvatura de las columnas, se tomó como el promedio de las cargas axiales máxima y mínima obtenidas del análisis estructural ante la acción sísmica de diseño correspondiente al ELCP, de acuerdo a lo recomendado por Rodríguez (2013). (4) El ancho de la losa que interactúa con las vigas, tanto en momento flexionante negativo como positivo, se definió a partir de las recomendaciones de Pantazopoulou y French (2001), para una distorsión de entrepiso de 2\%.

El momento de fluencia de una sección $\left(M_{y}\right)$, y su correspondiente curvatura, $\varphi_{y}$, se definieron como el momento y la curvatura correspondientes a cuando alguna barra de refuerzo longitudinal alcanza su esfuerzo de fluencia. La curvatura última de una sección $\left(\varphi_{u}\right)$ se definió como la menor curvatura 
correspondiente a las dos condiciones siguientes: (1) El concreto ubicado en la fibra exterior de la sección alcanza su deformación unitaria última a compresión, o de aplastamiento, $\varepsilon c_{u}=0.004$. (2) Una de las barras del refuerzo longitudinal a tensión alcanza su deformación unitaria última, o de fractura. Cabe notar que en el caso de que rija la primera de estas condiciones, el estado límite de las secciones no corresponde a un estado de falla, sino a un estado límite de utilidad. El momento último de la sección $\left(M_{u}\right)$ es el correspondiente a $\varphi_{u}$. La primera rama del diagrama momento-curvatura bilineal idealizado de una sección se define por medio de una línea recta que conecta el origen con el punto definido por $\left(M_{y}, \varphi_{y}\right)$. La segunda rama del diagrama idealizado se define por medio de una línea recta que conecta el punto anterior con el definido por $\left(M_{u}, \varphi_{u}\right)$.La pendiente de la primera rama del diagrama idealizado define la rigidez efectiva a flexión inicial de la sección transversal $\left(E I_{\text {eff }}\right)$, donde $E$ es el módulo de elasticidad del concreto e $I_{\text {eff }}$ es el momento de inercia efectivo inicial de la sección transversal. De forma similar, la pendiente de la segunda rama del diagrama idealizado, define la rigidez de posfluencia efectiva a flexión de la sección transversal $\left(E I_{\text {effpos }}\right)$, la cual se expresa como una fracción $(\alpha)$ de $E I_{\text {eff, }}$ es decir: $E I_{\text {effpos }}=\alpha E I_{\text {eff. }}$ Es importante mencionar que el criterio adoptado para caracterizar la rigidez efectiva inicial de una sección, se debe a que en las dos estructuras, la acción sísmica de diseño que menores demandas de resistencia impone a los miembros, son cercanas a las correspondientes a su fluencia incipiente.

Para cada sección transversal extrema de una viga se calcularon dos diagramas momento-curvatura y sus correspondientes idealizaciones bilineales, uno a momento flexionante negativo y otro a momento flexionante positivo. El $I_{\text {eff }}$ representativo de una sección extrema de viga se obtiene como el promedio de los obtenidos de los diagramas a momento negativo y positivo. De forma similar, el $I_{\text {effpos }}$ representativo de una sección extrema de viga, se expresa en función del valor promedio de los parámetros $\alpha$ obtenidos de los diagramas a momento negativo y positivo. El $I_{\text {eff }}$ de una viga se obtiene de manera simplista, como el promedio $\left(I_{\text {eff } \_ \text {rom }}\right)$ de los $I_{\text {eff }}$ representativos de sus secciones extremas. En la tabla 3 se muestran los $I_{\text {eff_prom }}$ de las vigas, normalizados por el momento de inercia de la sección transversal bruta de concreto $\left(I_{g}\right)$. Para obtener un valor representativo del parámetro $\alpha$ en vigas se aplicó un procedimiento análogo al anterior, obteniéndose así los $\alpha_{\text {prom }}$ mostrados en la tabla 3. Puesto que el momento resistente de las secciones transversales extremas de las columnas en ambos sentidos es el mismo, el $I_{\text {eff }}$ y el parámetro $\alpha$ de una columna se obtienen; también de manera simplista, a partir del diagrama momento-curvatura idealizado de una de sus secciones transversales extremas. En la tabla 3 se muestran los valores de estos parámetros. Cada uno de los puntos que definen el diagrama de interacción de las columnas, están asociados a la fluencia incipiente del acero de refuerzo.

Tabla 3. Algunas propiedades mecánicas de los miembros estructurales de los marcos estudiados.

\begin{tabular}{|c|c|c|c|c|c|c|c|c|c|}
\hline & \multirow[b]{2}{*}{ Nivel } & \multicolumn{4}{|c|}{ marco E1 } & \multicolumn{4}{|c|}{ marco E2 } \\
\hline & & $I_{\text {eff_prom }} / I_{g}$ & $\alpha_{\text {prom }}$ & $\theta_{u}^{-}$ & $\theta_{u}^{+}$ & $I_{\text {eff_prom }} / I_{g}$ & $\alpha_{\text {prom }}$ & $\theta_{u}^{-}$ & $\theta_{u}^{+}$ \\
\hline \multirow{3}{*}{ 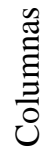 } & 1 a 5 & $0.39-0.43$ & $0.046-0.058$ & ---- & $0.010-0.014$ & $0.45-0.49$ & $0.057-0.075$ & ---- & $0.010-0.013$ \\
\hline & 6 a 10 & $0.35-0.40$ & $0.054-0.065$ & ---- & $0.009-0.012$ & $0.37-0.43$ & $0.054-0.065$ & ---- & $0.009-0.012$ \\
\hline & 11 a 15 & $0.33-0.38$ & $0.044-0.054$ & ---- & $0.011-0.015$ & $0.34-0.39$ & $0.044-0.054$ & ---- & $0.011-0.015$ \\
\hline \multirow{3}{*}{$\stackrel{\infty}{=}$} & 1 a 8 & 0.48 & 0.020 & 0.024 & 0.049 & 0.70 & 0.022 & 0.023 & 0.047 \\
\hline & 9 a 12 & 0.47 & 0.018 & 0.021 & 0.050 & 0.60 & 0.020 & 0.022 & 0.046 \\
\hline & 13 a 15 & 0.38 & 0.019 & 0.024 & 0.049 & 0.45 & 0.020 & 0.024 & 0.050 \\
\hline
\end{tabular}

La capacidad rotacional última de las secciones extremas de los elementos estructurales se obtuvo por medio de la ec. 13:

$\theta_{u}=\theta_{y}+\left(\varphi_{u}-\varphi_{y}\right) L p$ 
donde $\theta_{u}$ es la capacidad última de rotación de la sección, $\theta_{y}$ es su rotación de fluencia y $L p$ es la longitud equivalente de plastificación de la articulación plástica, supuesta como la mitad del peralte total para las columnas y como $2 / 3$ de dicho peralte para vigas.

Para las secciones extremas de las vigas se calcularon las capacidades rotacionales a momento negativo $\left(\theta_{u^{-}}\right)$y a momento positivo $\left(\theta_{u}+\right)$, y para el caso de las columnas un único valor, $\theta_{u}$. En la tabla 3 se muestran los valores de las capacidades rotacionales de los elementos estructurales.

\section{Consideraciones para los análisis pushover}

En los modelos analíticos de las estructuras para realizar los análisis pushover se utilizaron las propiedades de los miembros estructurales previamente calculadas. Se consideró además que las articulaciones plásticas en vigas y columnas tienen el mismo valor de $L p$ usado para caracterizar su capacidad rotacional. También, al igual que en el diseño, se consideraron los efectos $P-\triangle$ y se ignoró la interacción suelo estructura. La capacidad de deformación lateral última de los marcos se definió como la correspondiente a la etapa de carga en la que todas las vigas de algún entrepiso alcanzan su capacidad rotacional última.

Actualmente se reconoce que el uso de un análisis pushover convencional, en el cual el patrón de fuerzas laterales es función de las propiedades dinámicas modales de la estructura en su estado elástico; y permanece invariante durante su respuesta inelástica, "obliga" a una estructura a responder en una forma que puede diferir significativamente de la forma en la que responderá durante su respuesta inelástica. Para evitar esta posible limitante, en el presente estudio se utilizó una variante mejorada de este tipo de análisis estático inelástico, conocida como pushover adaptable. En esta variante, el patrón de fuerzas laterales es función de las propiedades dinámicas modales de la estructura en cada etapa de su respuesta inelástica, comúnmente denominadas en la literatura como propiedades dinámicas "instantáneas". En particular, se utilizó el pushover adaptable implementado en el programa Ruaumoko 2D (Carr, 2000).

\section{$\underline{\text { Resultados de los análisis pushover }}$}

Los periodos fundamentales elásticos de los marcos E1 y E2 son, $1.90 \mathrm{~s}$ y $1.66 \mathrm{~s}$ respectivamente, los cuales son: mayor, y aproximadamente igual a los estimados en el diseño. Para el caso del marco E1, este resultado se debe a que, mientras las relaciones $I_{\text {eff }} I_{g}$ estimadas para las vigas son similares a las estipuladas por las NTCC-2004 $\left(I_{e f f} / I_{g}=0.5\right)$, dichas relaciones estimadas para columnas son significativamente menores a las estipuladas por las normas $\left(I_{\text {eff }} / I_{g}=1.0\right)$, como se aprecia en la tabla 3 . Para el caso del marco E2, este resultado se debe a que, mientras las relaciones $I_{e f f} / I_{g}$ estimadas para las vigas son moderadamente mayores a las estipuladas, dichas relaciones estimadas para columnas son significativamente menores a las estipuladas, como se aprecia en la tabla 3, lo que resultó en una "compensación" de la rigidez lateral del marco. El hecho de que el periodo del marco E2 sea menor que el del marco E1, se debe a que la mayoría de los miembros estructurales del marco E2 tienen mayor cuantía de refuerzo que los correspondientes del marco E1.

En las figs. 10a y 11a se muestran las relaciones cortante basal versus distorsión de entrepiso máxima de los marcos E1 y E2, respectivamente. En la fig. 10a se observa que, debido a las fuentes de sobrerresistencia consideradas, el cortante basal asociado a la fluencia incipiente $\left(V b_{F I}\right)$ del marco E1, que es el correspondiente al desarrollo de la primera articulación plástica en la sección extrema de algún miembro estructural, es mayor al $V b_{D}$. En la misma figura también se observa que $V b_{F I}$ es menor a $V b_{E L S}$, lo que indica que cuando la estructura desarrolla el $V b_{E L S}$, ya ha ingresado en su intervalo de comportamiento inelástico y debido a esto, su rigidez lateral ha disminuido, razón por la cual, la máxima distorsión de entrepiso correspondiente es considerablemente mayor a la permisible para el ELS. Estos 
resultados muestran que si se asigna a una estructura un $V b_{D}$ menor a $V b_{E L S}$, no se cumplirán los parámetros de desempeño requeridos para el ELS.

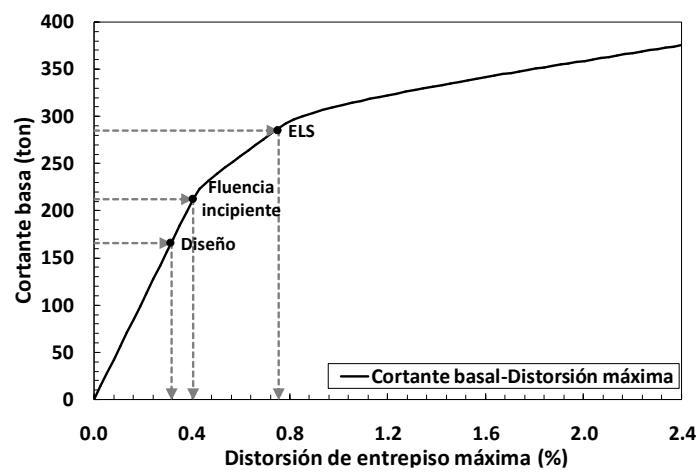

a). Cortante basal versus distorsión de entrepiso máxima

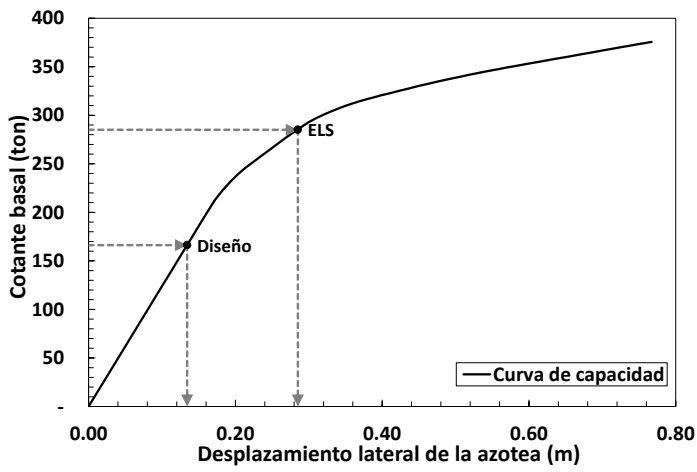

b). Cortante basal versus desplazamiento de azotea

Figura 10. Relaciones cortante basal versus deformaciones laterales máximas del marco E1

En la fig. 11a se observa que el $V b_{F I}$ del marco E2 es mayor que $V b_{D}$ y $V b_{E L S}$, por lo que el comportamiento de la estructura ante la acción sísmica de diseño correspondiente al ELS será elástico. También se observa que debido a que el periodo fundamental elástico del marco E2 obtenido del análisis pushover es ligeramente mayor al de diseño, la máxima distorsión correspondiente al $V b_{E L S}(0.466 \%)$, es también ligeramente mayor a la de diseño $(0.427 \%)$. Estos resultados muestran que si se asigna a una estructura un $V b_{D}$ aproximadamente igual $V b_{E L S}$, se cumplirán los parámetros de desempeño requeridos para el ELS.

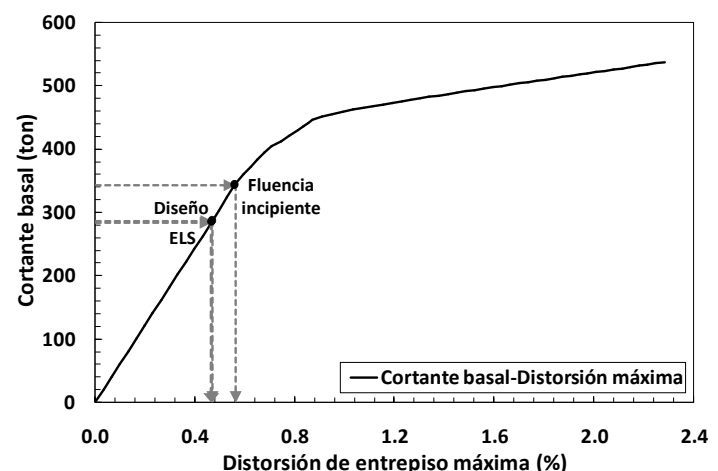

a). Cortante basal versus distorsión de entrepiso máxima

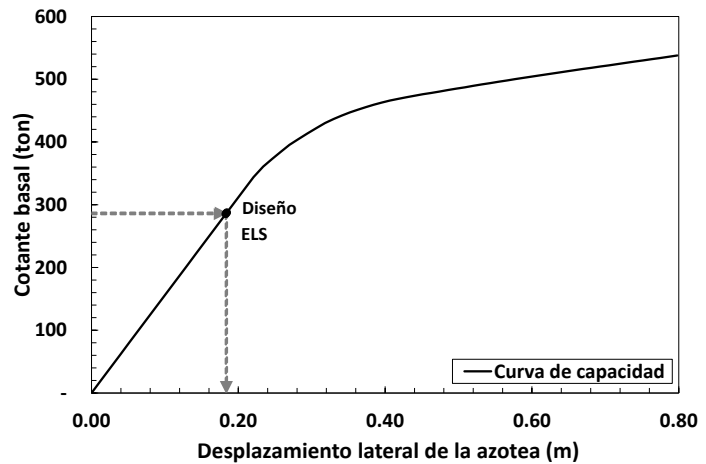

b). Cortante basal versus desplazamiento de azotea

Figura 11. Relaciones cortante basal versus deformaciones laterales máximas del marco E2

Como información complementaria a la presentada en las figs. 10a y 11a, en las figs. 10b y $11 \mathrm{~b}$ se muestran las gráficas de las relaciones cortante basal-desplazamiento lateral máximo de la azotea. Estas relaciones comúnmente se conocen como curvas de capacidad de una estructura. En estas curvas se observan los mismos resultados que en las curvas de las figs. 10a y 11a. También, como comentario complementario, si los resultados obtenidos de los análisis pushover se interpretan únicamente en términos de distorsión, de la fig. 10 se observa que para la distorsión de entrepiso permisible para el ELS adoptada en este estudio (0.4\%), el marco E1 se comporta elásticamente, por lo que podría pensarse que 
su desempeño es adecuado. Sin embargo, esta impresión es errónea, ya que el cortante basal correspondiente a la distorsión de $0.4 \%$ es considerablemente menor al $V b_{E L S}$.

\section{CONCLUSIONES Y RECOMENDACIONES}

En este estudio se mostró que las estructuras ubicadas en sitios del valle de México con $T s$ mayores a $1.65 \mathrm{~s}$; y diseñadas para los valores de $Q$ estipulados en las NTCS-2004 para estructuras dúctiles, responden inelásticamente al sismo de diseño correspondiente al ELS, y por lo tanto no cumplen los parámetros de desempeño establecidos en el AA de dichas normas.

Con base en los aspectos discutidos y los resultados mostrados en este estudio, se hacen las siguientes recomendaciones para el diseño sísmico de estructuras a ubicarse en estos sitios:

I. $\quad$ Por sencillez, diseñar para $Q=2$.

II. Si por razones económicas se desea reducir las fuerzas sísmicas elásticas de diseño al máximo, se recomienda definir el cortante basal de diseño con el espectro reducido por $Q^{\prime}\left(Q_{\max }\right)$ y $R$.

III. Independientemente del valor máximo de $Q$ con el que se desee o se deba diseñar, aplicar los requisitos establecidos por las NTCC-2004 para el diseño y construcción de estructuras dúctiles.

\section{AGRADECIMIENTOS}

El primer autor agradece al Consejo Nacional de Ciencia y Tecnología la beca otorgada para la realización de sus estudios de posgrado. También se agradecen las observaciones y comentarios de Saúl López y Manuel Pinzón, así como las de los revisores anónimos de este artículo.

\section{REFERENCIAS}

Carr, A. J. (2000), "Ruaumoko 2D, inelastic dynamic analysis computer program and users manual", University of Canterbury, Department of Civil Engineering, Nueva Zelanda.

CSI (2010), "SAP2000 v.14, integrated finite element analysis and design of structures", Computer and Structures Inc., Berkeley, California, Estados Unidos.

Mander, J. B., M. J. N. Priestley y R. Park (1988), “Theorical Stress-Strain Model for Confined Concrete", Journal of Structural Engineering, ASCE, Vol. 114, No. 8, pp. 1804-1826.

Mayes, R. L. (1995), "Interstory drift design and damage control issues", The Structural Design of Tall buildings, Vol.4, pp. 15-25.

NTCC-2004 (2004), "Normas Técnicas Complementarias para Diseño y Construcción de Estructuras de Concreto", Gaceta Oficial del Distrito Federal.

NTCS-2004 (2004), “Normas Técnicas Complementarias para Diseño por Sismo”, Gaceta Oficial del Distrito Federal.

Ordaz, M., E. Miranda y J. Avilés (2003), "Propuesta de espectros de diseño por sismo para el D.F.", Revista Internacional de Ingeniería de Estructuras, Vol. 8, No. 2, pp. 189-207. 
Pantazopoulou, S. J. y C. W. French (2001), “ Slab participation in practical earthquake design of reinforced concrete frames", ACI Structural Journal, Vol. 98, No. 4, pp. 479-489.

Rodríguez, M. y J. C. Botero (1996), "Aspectos del comportamiento sísmico de estructuras de concreto reforzado considerando las propiedades mecánicas de aceros de refuerzo producidos en México", Publicación Series del Instituto de Ingeniería, UNAM, No. 575.

Rodríguez, M. y J. I. Restrepo (2012), "Práctica y diseño sísmico de edificios en México-cambios necesarios", Revista de Ingeniería Sísmica, No. 86, pp. 89-118.

Rodríguez, M. (2013), “Comunicación personal”, Instituto de Ingeniería, UNAM, México.

Uang, C. (1991), "Establishing R (or Rw) and Cd Factors for Buildings Seismic Provisions", Journal of Structural Engineering, Vol. 117, No.1. 